Rev. Adm. Saúde (On-line), São Paulo, v. 21, n. 83: e291, abr. - jun. 2021, Epub 06 jul. 2021 http://dx.doi.org/10.23973/ras.83.291

RELATO DE CASO

\title{
Proposta de modelo assistencial para uma operadora de saúde suplementar em expansão na cidade de São Paulo
}

Proposal for an assistance model for an expanding supplementary health company in the city of São Paulo

\section{Lais de Sá Fonseca ${ }^{1}$, Alberto Jose Niituma Ogata²}

\section{Mestre em gestão. Escola de Administração de Empresas da Fundação Getúlio Vargas} (EASP/FGV), São Paulo SP

2. Doutor em saúde coletiva. Pesquisador associado do Centro de Estudos em Planejamento e Gestão de Saúde da EAESP/FGV, São Paulo SP

\section{RESUMO}

Introdução: O sistema de saúde suplementar enfrenta atualmente, assim como o setor público, os efeitos do aumento dos custos assistenciais. O setor saúde é influenciado pelo cenário econômico e sua atividade contribui com a geração de empregos e renda, e estimula o crescimento em investimentos e capital. O incremento das despesas neste segmento, está associado ao envelhecimento da população, às mudanças no perfil de consumo dos produtos de saúde, à incorporação de novas tecnologias, à falta de eficiência das ações preventivas e aos modelos de remuneração. Dessa forma, as operadoras passam a transformar as maneiras de ofertar cuidados através de novas diretrizes que buscam otimizar recursos e obter melhores resultados assistenciais, e assim, sustentabilidade no mercado de saúde privado.

Objetivos: O presente trabalho visa propor a uma operadora de saúde privada em expansão de atividades um modelo assistencial baseado em referenciais teóricos e nas práticas do mercado de saúde suplementar atual. Método: 
Trata-se de pesquisa qualitativa com estratégia de estudo de caso. A análise descreve a operadora e suas características e detalha opções de modelo assistencial a serem aplicados em função da expansão do número de beneficiários. Resultados: A proposta de modelo para abordagem assistencial incluiu o foco em estrutura de atenção primária, a implantação de unidades de transição e cuidados paliativos e a prática de saúde integrativa. Conclusões: O estudo propõe alternativas para inserir o paciente no centro do cuidado e buscar melhor experiência, valor e fidelidade do cliente. A proximidade de equipe multidisciplinar exclusiva aos pacientes favorece, aliada ao segmento de diretrizes clínicas de indicadores processuais, o monitoramento sobre a utilização dos serviços disponíveis no sistema e a avaliação dos resultados operacionais.

Palavras-chaves: Saúde Suplementar, Gestão em Saúde, Governança Clínica, Planejamento em Saúde, Sistemas de Saúde, Doenças Crônicas.

\begin{abstract}
Introduction: The supplementary health system is currently facing, as well as the public system, the effects of rising health care costs. The health sector is affected by the economic scenario and its activity contributes to generation of jobs, income, and stimulates growth in investments and capital. The increase in expenses in this segment is associated with ageing of population, changes in the consumption profile of health products, the incorporation of new technologies, the lack of efficiency in preventive actions and payment models. Thus, health companies begin to transform the ways of offering care through new guidelines that seek to optimize resources and obtain better care results, and thus, sustainability in the private health market. Objectives: The present study aims to propose to a private health operator in expansion of activities a care model based on theoretical references and on the practices of the current supplementary health market. Method: It is a qualitative research with a case study strategy. The analysis describes the company and its characteristics and details care model options to be applied due to the expansion in the number of customers. Results: The proposed model for the care approach included the focus on primary care structure, the implementation of transition units and palliative care and the practice of integrative health. Conclusions: The study proposes alternatives to insert patient centered care and seek better experience, value and customer loyalty. The proximity of an exclusive multidisciplinary team to patients favors, together with the segment of clinical guidelines for procedural indicators, the monitoring of the use of services available in the system and the evaluation of operational results.
\end{abstract}

Keywords: Supplemental Health. Health management. Clinical Governance. Health Planning. Health Systems. Chronic Diseases. 


\section{INTRODUÇÃO}

O planejamento estratégico organizacional é de fundamental importância para a conquista do equilíbrio entre o propósito de uma instituição e a expectativa dos clientes aos quais se destinam suas atividades. Na área da saúde, esta relação se demonstra complexa em virtude de ambiente externo de grande competitividade, concomitante a um cenário repleto de transformações, ameaças, e, simultaneamente, grandes oportunidades de negócios ${ }^{(1)}$.

O processo de inovação institucional permite a criação de novos métodos, produtos e atividades, é fundamental para a adaptação às mudanças de mercado e se aproximam ao cliente, objetivo principal. A saúde tem a inovação como aliada, visto que esta permite o desenvolvimento dos recursos necessários para o crescimento de propostas que se somam às anteriormente praticadas. Traz consigo incremento nos custos, porém permite assistência de melhor qualidade e maior possibilidade de enfrentamento da competitividade.

As operadoras de saúde suplementar ofertam assistência privada à saúde atuando em oito diferentes modalidades. A atenção à reorganização dos modelos de assistência é um objetivo comum neste mercado, dadas as previsões das mudanças relacionadas ao perfil epidemiológico populacional e demográfico, além dos hábitos de vida. Cada modalidade deve garantir o acesso e a assistência à saúde de seus usuários, e, nesse sentido, há a definição e aplicação de regras pelo Estado através da Agência Nacional de Saúde Suplementar (ANS). Este órgão regulatório monitora as ações das operadoras buscando a validação do controle adequado entre a demanda e oferta de serviços, com cumprimento das obrigações perante a responsabilidade social e do papel das empresas no mercado de saúde (2).

Atualmente, os estudos e previsões relacionados à carga global de doenças, seus impactos e relações com fatores de risco, associados à necessidade do cuidado contínuo das doenças crônicas, vão de encontro aos quatro objetivos principais da gestão em saúde: melhorar a experiência dos pacientes com os serviços; aprimorar a saúde da população; reduzir os custos e aperfeiçoar a experiência dos profissionais deste segmento ${ }^{(3)}$.

A referência das práticas de saúde nos últimos anos demonstra assistência fragmentada, direcionada ao uso excessivo de procedimentos e especialidades, cuidado desintegrado e descentralizado. Estes padrões resultam em ineficiência e desfechos desfavoráveis, pois, são atrelados à demanda espontânea e utilização inapropriada. Nesse contexto, a Assistência Primária à Saúde (APS) está sendo considerada pelas operadoras, pois, vem contribuir para a maior equidade nos serviços. Traz a possibilidade de reduzir diferenças entre grupos populacionais, promovendo o cuidado coordenado, individualizado, focado na pessoa e não na doença, e integrado com as necessidades de saúde, desde a prevenção até a reabilitação. Esta estrutura traz maior satisfação dos clientes, porém, exige adaptação conforme o perfil heterogêneo da carteira, baseada nas informações referentes à população envolvida (4). 
A incidência da carga global de doenças no Brasil, quando analisado o seu impacto nas unidades federadas, correlaciona-se aos fatores de risco e à mensuração da fração atribuível para as causas de morte e para os anos de vida perdidos da população, como também aos anos de vida vividos com incapacidades. Esta explanação justifica a necessidade de atividades requeridas de proteção e promoção à saúde, além da prevenção e gerenciamento de doenças crônicas. A quantificação dos impactos sobre a saúde de um conjunto de fatores de risco é uma contribuição importante para melhorar a saúde da população ${ }^{(5)}$.

Os dados populacionais do Brasil evidenciam o avanço do envelhecimento da população, com a projeção do aumento no número de idosos (pessoas com 60 anos de idade ou mais) que em 2017 somavam 14\% da população para 34\% previstos para 2060 . ${ }^{(6)}$. As condições crônicas traduzem $75 \%$ da carga global brasileira. É preciso haver harmonia entre as condições de saúde e as instituições assistenciais. O desencontro observado ao se atender situações de doenças crônicas e agudas com a mesma metodologia traz instabilidade no controle de efetividade da saúde em que não se observa a comunicação esperada entre os diferentes níveis de assistência, apenas ações reacionárias às demandas de doenças, sem percepções de reações de autocuidado (7).

As doenças crônicas têm grande impacto sobre as causas de morte e causam grandes restrições às atividades de lazer e ocupações. As operadoras não têm conhecimento sobre a saúde dos seus beneficiários e a utilização dos serviços. Somente observa-se a existência de informações sobre questões financeiras relacionadas às despesas com os procedimentos. Desse modo, é fundamental que as empresas de saúde privada utilizem os recursos disponíveis para gerenciar a saúde dos seus usuários. $O$ cuidado integrado abrange $o$ envolvimento das pessoas com a responsabilidade do autocuidado e pode ser atingido com ações de promoção e prevenção propostas pela operadora. Tais ações necessitam abordar inclusive a saúde mental, evidenciada pelo número crescente de utilização de serviços de atenção psiquiátrica e emocional (2).

Ainda no que se relaciona ao envelhecimento, o número estimado de novos casos de câncer no Brasil de ambos os sexos e todas as idades irá aumentar em 78,5\% entre 2018 e 2040 , de 559.371 novos casos em 2018 para 998.056 casos em $2040{ }^{(8)}$. A oncologia integrativa se define como cuidado à saúde que inclui um balanço entre as abordagens de mudanças comportamentais, estilos de vida, e o uso de terapias complementares que se associam aos tratamentos convencionais, de forma individualizada e segura, baseada nas informações obtidas através de entrevistas abrangentes. Os pacientes buscam por estas oportunidades de tratamento, para obterem melhorias do quadro físico, emocional e bem-estar, redução do quadro álgico e outros sintomas ${ }^{\left({ }^{(9)}\right.}$.

Entre 2013 e 2018, apesar da redução no número de beneficiários de planos de assistência médico-hospitalar em 3,2\%, (de 48,7 milhões para 47,2 milhões), houve aumento no número de procedimentos em $5,4 \%$ (1,11 bilhão para 1,4 bilhão) e, consequentemente, das despesas assistenciais em expressivos 74\% (92 bilhões em 2013 para 160 bilhões em 2018). Tais procedimentos são relacionados na maior proporção a exames, seguidos por consultas ambulatoriais, outros atendimentos ambulatoriais, terapias, consultas 
em pronto-socorro, e internações. A maior proporção de despesas assistenciais se justifica pelas internações, seguidas por exames, consultas ambulatoriais, outros atendimentos ambulatoriais, terapias, demais despesas e consultas em pronto-socorro ${ }^{(10)}$.

O sistema de saúde suplementar neste contexto é complexo, o que implica na necessidade de diversas estratégias corporativas que visam manter a universalização do direito social à saúde e encontra a dificuldade em enfrentar custos dispendiosos operacionais ${ }^{(11)}$.

Tal esforço se mostra indispensável, visto que os fatores econômicos associados à transição demográfica, epidemiológica e ao fato de novas tecnologias e recursos se tornarem cada vez mais dispendiosos, poderem resultar na insolvência das operadoras de saúde. Portanto, previsões futuras relacionadas à economia em saúde devem se associar diretamente aos custos gerados por condições crônicas ${ }^{(12)}$.

Diante de tamanha complexidade e risco no que diz respeito à sustentabilidade financeira das empresas de saúde, a satisfação do paciente não pode ser desconsiderada. E importante relacionar as diferentes abordagens de melhorias executadas por organizações em saúde buscando eficiência, ao significado de valor para os usuários dos serviços. A visão de valor tem diferentes significados dentro da cadeia que envolve os sistemas de saúde, e a sua classificação deve ser adotada e considerada para que resultados globais sejam alcançados ${ }^{(13)}$.

Como fator associado a este cenário, está a exposição dos pontos relevantes para a formação e organização das redes de atenção à saúde: a importância na definição da atenção primária na abrangência de cada região; sua capacidade de absorção e principalmente a integração com os outros níveis de assistência, a atenção às doenças crônicas e à assistência hospitalar (14).

Deste modo, analisam-se as estratégias que a saúde suplementar pode propor, como a organização da gestão, a identificação do perfil de risco, o foco nos programas preventivos e de autocuidado, além das maneiras de recompensar os beneficiários contemplando-os com alguns benefícios quando comprovada a sua adesão à prevenção ${ }^{(15)}$.

A definição redes de atenção à saúde estabelece organização coordenada que promove assistência de população conhecida, com princípios de integralidade e horizontalidade. Considera a complexidade do cuidado nos níveis primários e a possibilidade em atender às demandas agudas conforme a necessidade, e o acompanhamento contínuo de casos crônicos ${ }^{(16)}$.

Organizações que buscam a verticalização dos serviços como diretriz, usufruem dos princípios de diversificação da atuação, o que traz capacidade de desenvolvimento de novos recursos e atividades que possam gerar estratégias competitivas em ambientes que elas dominem ${ }^{(1)}$.

Diante de tais desafios e cenário, decidimos realizar estudo de caso de uma operadora de saúde suplementar, buscar alternativas e propor soluções para a 
expansão de suas atividades. A abordagem baseada em modelo assistencial procura atender às perspectivas de conhecimento dos grupos de clientes, oferta de cuidado coordenado e obtenção de resultados satisfatórios e sustentáveis.

\section{MÉTODO}

Trata-se de pesquisa qualitativa com estratégia de estudo de caso, que se caracteriza pela coleta e avaliação de dados.

A aplicação deste método permite abordagens específicas que contribuem com o planejamento de projetos, pois, auxiliam a identificação de tendências e atitudes e despertam a reflexão do autor sobre possibilidades futuras relacionadas a determinados fenômenos ${ }^{(17)}$.

A proposta é de análise interpretativa do mercado privado de saúde, no que diz respeito à atuação dos planos de saúde em relação aos desafios, estratégias comerciais, redução de custos e eficiência operacional.

Como métodos de coleta de dados foram realizados a revisão de literatura; coleta de dados da operadora fornecidos ao autor mediante autorização expressa da empresa e consulta à Sala de Situação da ANS, além de pesquisa de dados indicadores de saúde das regiões a serem inseridas como hipótese de atuação.

A revisão bibliográfica contempla as seguintes bases de dados: PubMed, MEDLINE, EBSCO, SciELO, LILACS e Google Acadêmico, através das palavras-chaves: "Saúde Suplementar", "Gestão em Saúde", "Governança Clínica", "Gestão em saúde", "Planejamento em Saúde", "Sistemas de Saúde" e "Doenças Crônicas".

A análise de indicadores será pesquisada nos sites dos órgãos: PROADESS; SEADE; PNAD e ANS. O estudo foi aprovado pelo Comitê de Conformidade Ética em Pesquisas Envolvendo Seres Humanos - CEPH/FGV, tendo sido dispensado de análise deste comitê.

\section{RESULTADOS}

\section{Estudo de Caso}

A empresa analisada é uma operadora privada de saúde, da modalidade filantrópica, que tem sua atuação restrita quase totalmente à cidade de São Paulo (SP). Oferece assistência à saúde nos níveis primário, secundário e terciário, através de unidades próprias hospitalar, ambulatoriais e centro de reabilitação, e rede referenciada hospitalar e ambulatorial. As atividades filantrópicas são realizadas através de parceiras entre a operadora e prefeituras de municípios do Estado de São Paulo. Os produtos comercializados contemplam assistência médica, hospitalar e odontológica. 


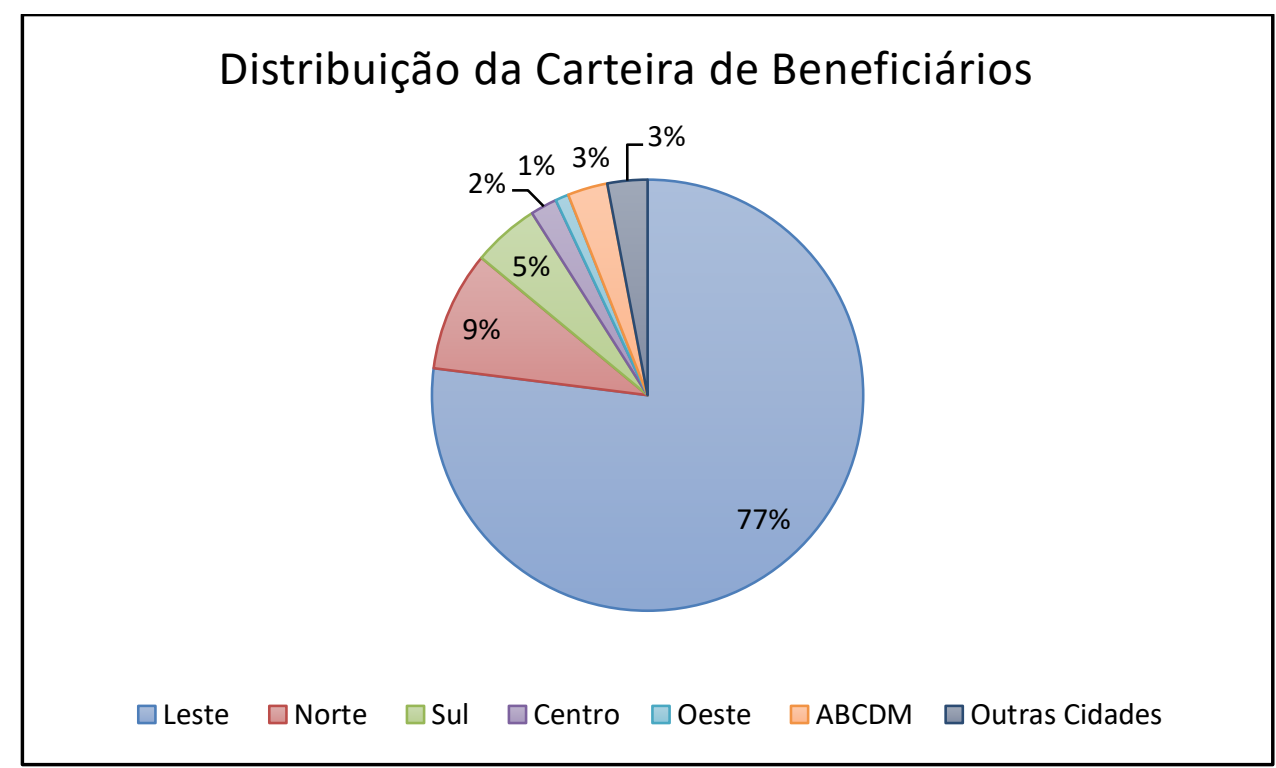

Gráfico 1. Distribuição carteira de beneficiários no município de São Paulo. Fonte: operadora.

\section{Faixa etária beneficiários}

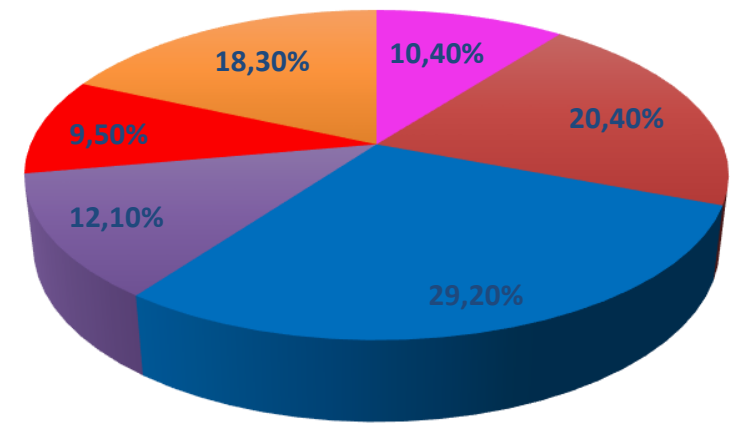

$\square$ a $14 \square 15$ a $29 \square 30$ a $44 \square 45$ a $59 \square 60$ a $74 \square$ acima de 75 anos

Gráfico 2: Faixa etária de beneficiários (dados referentes a fevereiro de 2019). Fonte: operadora.

Tabela 1. Número de beneficiários exclusivos por tipo de plano em novembro 2019. 


\begin{tabular}{l|l|l|l|l|l}
\hline & Variação no mês & & & Variação no mês & \\
120.194 & $-0,03 \%$ & $\downarrow$ & 2.199 & $10,84 \%$ & $\uparrow$ \\
\hline
\end{tabular}

Fonte: ANS

\section{Modelo Assistencial}

Com base no estudo da operadora, sua carteira de clientes e a decisão estratégica de expansão, propõe-se a adoção de um modelo de cuidado integral e integrado. A rede estrutural de assistência deve ser organizada de forma a abranger a população envolvida com atenção individualizada e acompanhada em todo o fluxo de atendimentos. As atividades assistenciais que promovam melhor experiência aos beneficiários com foco na geração de valor e retenção de clientes precisam estar ressaltadas.

Como suporte para o desenvolvimento da proposta, está a incorporação de tecnologia de informação, e demais serviços de apoio entre as unidades, que permitem a integração de informações e controle de indicadores de qualidade dos serviços. As estratégias de comunicação e divulgação da empresa, do mesmo modo, acompanham e auxiliam os objetivos de expansão. 


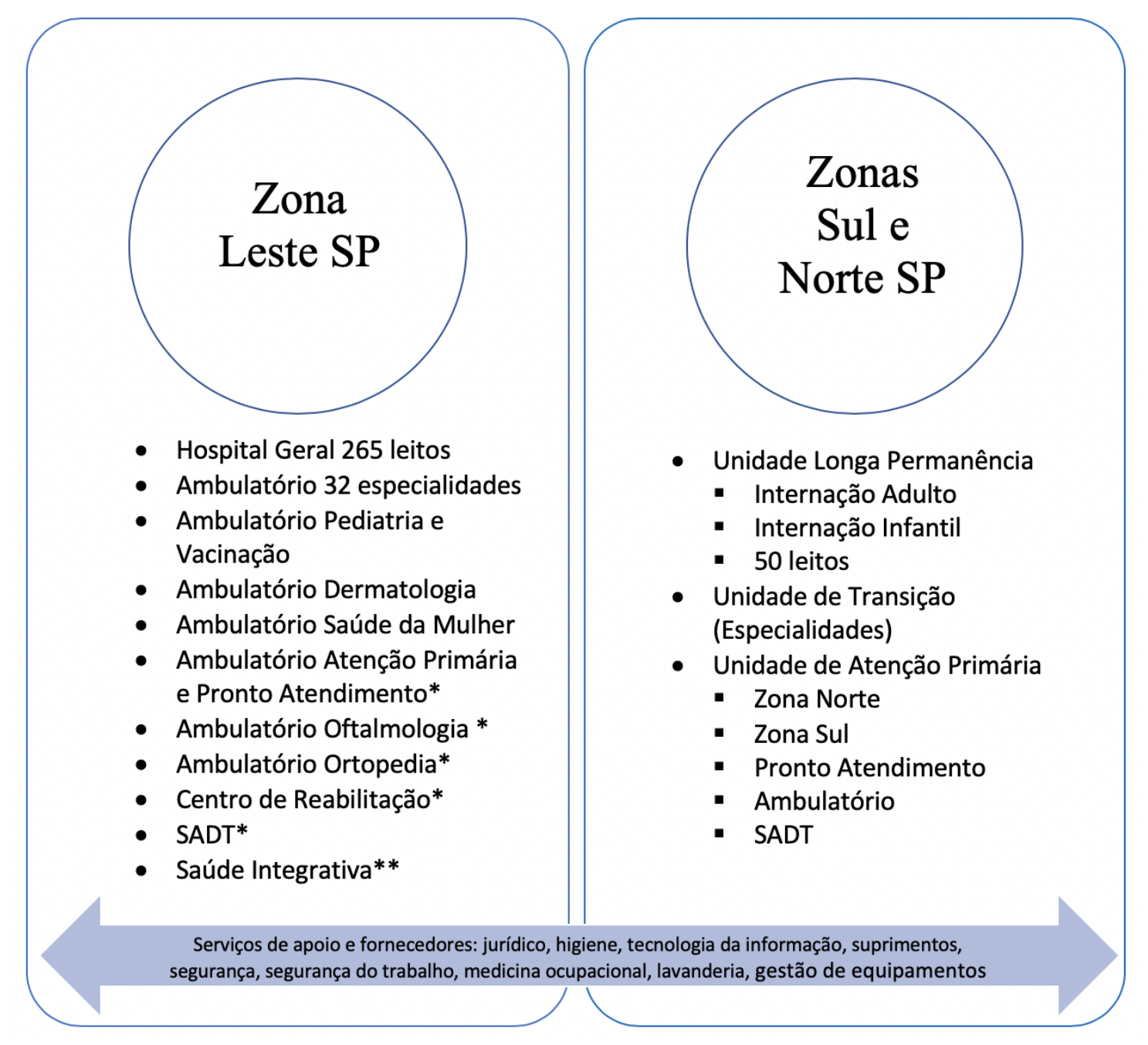

Figura 1. Unidades Assistenciais existentes na zona leste de São Paulo e proposta para novas unidades assistenciais para as regiões sul e norte.

Nota:* Unidades inauguradas pela operadora em 2019. ** Propõe-se a incorporação ao setor ambulatorial de especialidades. 


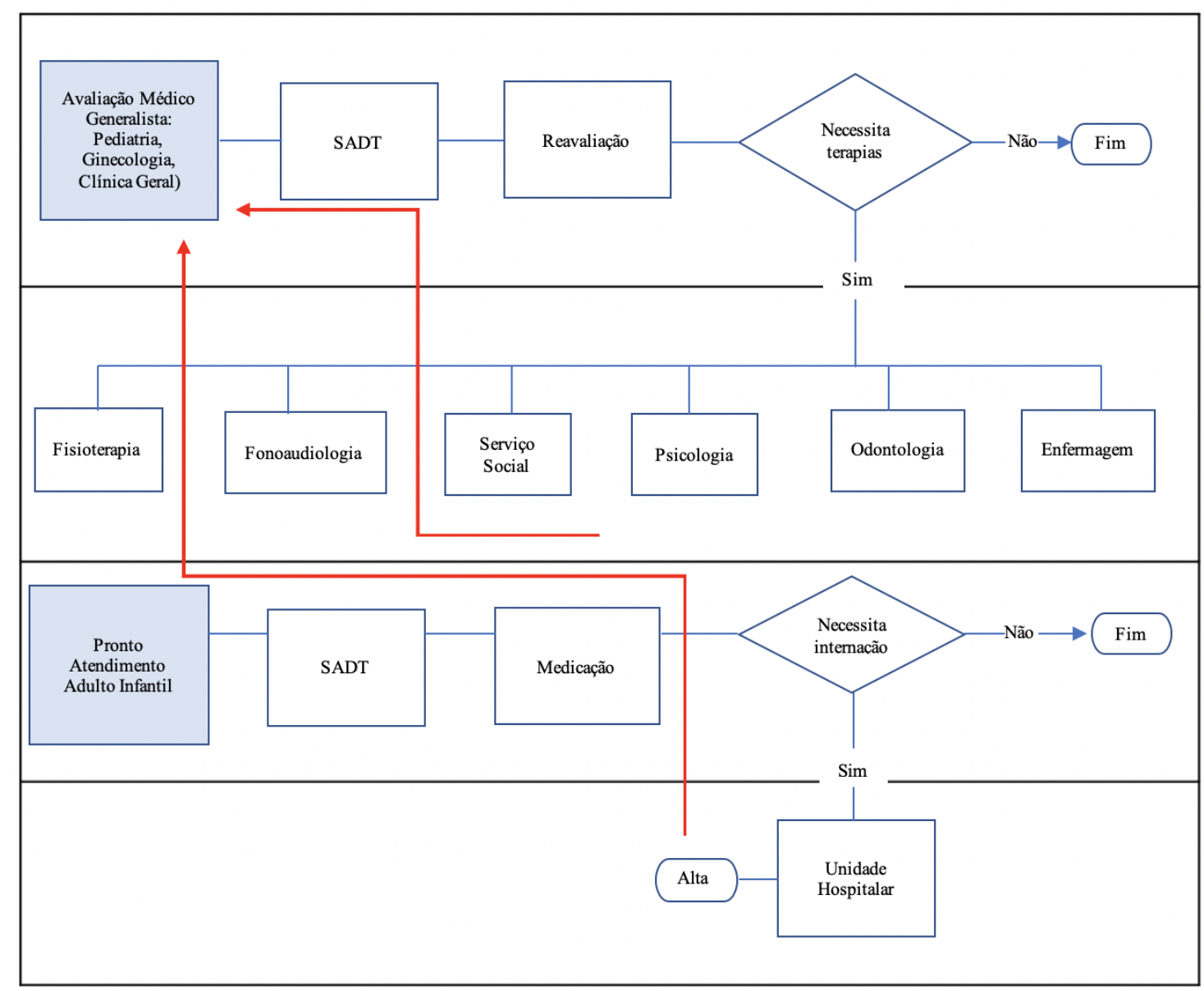

Figura 2. Fluxograma de atenção primária e contrarreferência. 


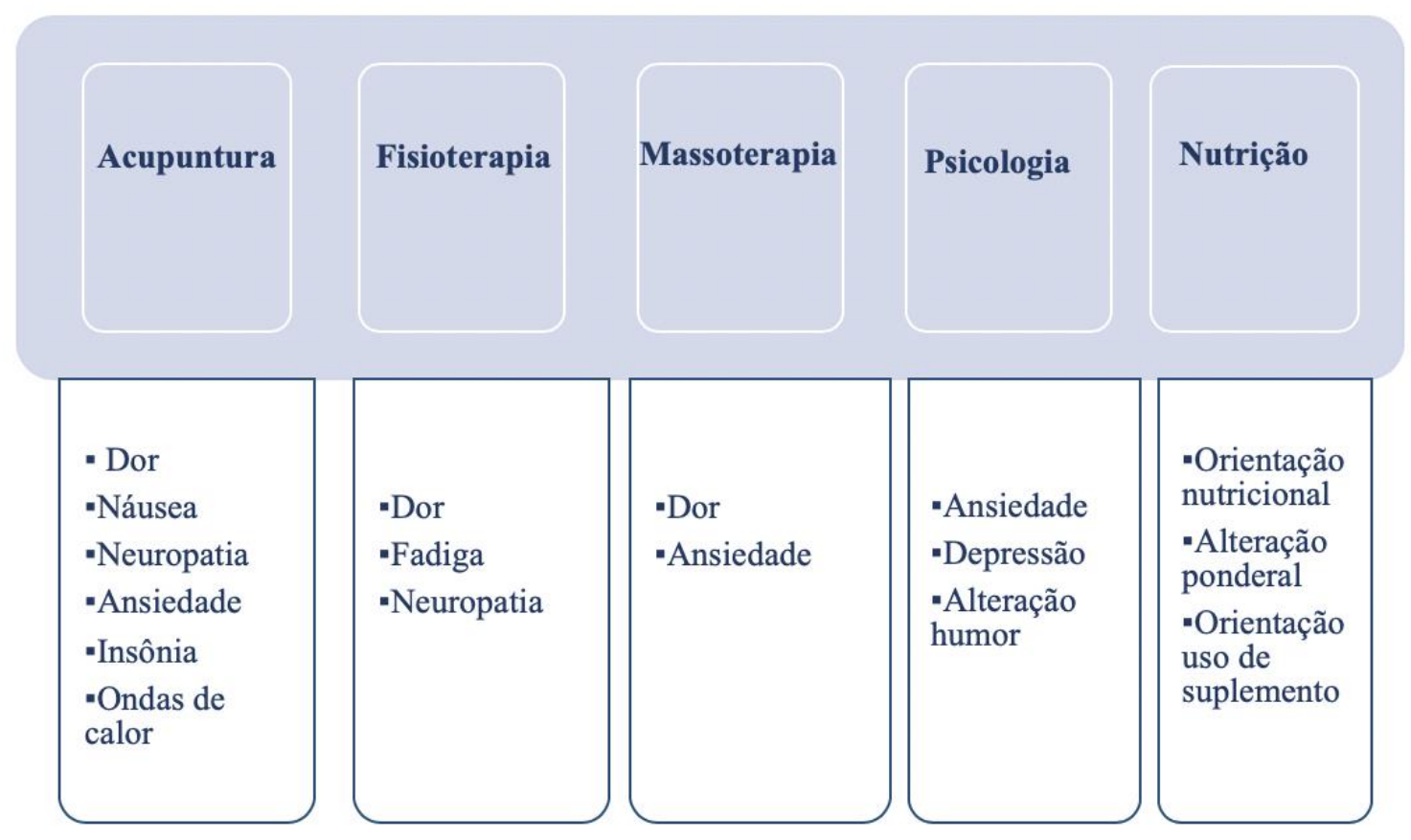

Figura 3. Profissionais da equipe de saúde integrativa e indicação de acompanhamento. 


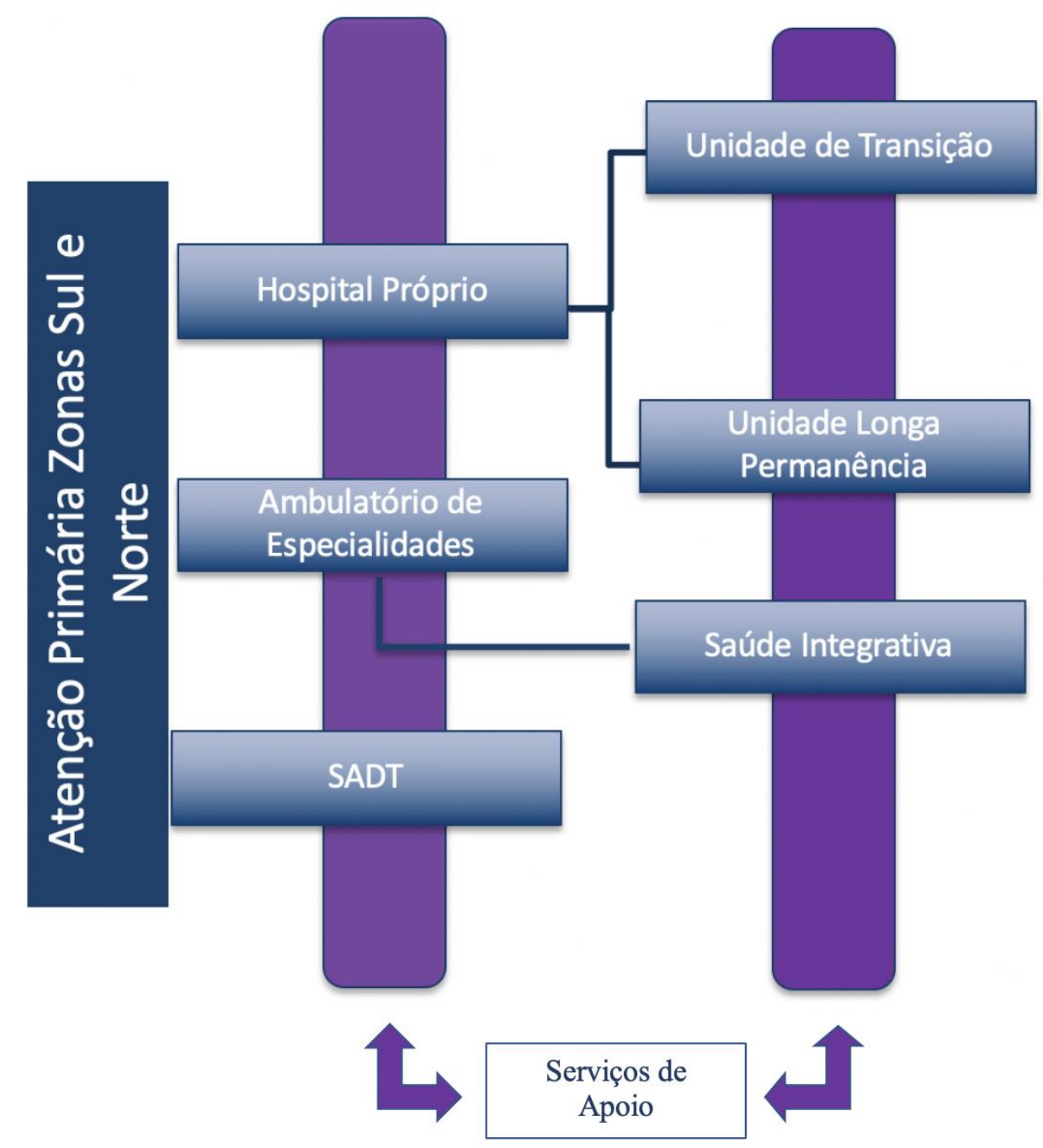

Figura 4. Integração entre unidades assistenciais.

\section{DISCUSSÃO}

O modelo assistencial das operadoras de saúde tem uma tendência de transição para propostas de atuação voltadas a atenção primária à saúde. Esta se destaca como ponto essencial no conjunto de elementos que formam a rede de atenção assistencial. Porém, o estabelecimento desta disposição não é simples, visto que requer a análise detalhada sobre as características da população incluída, assim como as particularidades de cada operadora, como serviços próprios ou referenciados, abrangência geográfica, além do porte, administração financeira e capacidade na integração de informações ${ }^{(4)}$.

O modelo de atenção primária “The Patient-Centered Medical Home”, enfatiza a coordenação do cuidado; processos de melhorias; tecnologia de informação, e a atribuição do cuidado individualizado a um profissional médico responsável pelo gerenciamento de cada paciente, assim como encaminhamentos aos 
prestadores necessários. Tem o objetivo de tratar necessidades agudas, porém, com o amplo propósito de melhorar o engajamento do paciente e implementar assistência baseada em evidências. Todas as metas são monitoradas e controladas conforme diretrizes de acreditação, e é de extrema importância identificar os principais fatores que levam a melhores desfechos e redução de custos. Modelos de pagamento alternativos são também praticados e essenciais para incentivar o controle de custos, como também para associálos às diretrizes baseadas em evidências e o gerenciamento da saúde populacional ${ }^{(18)}$.

A utilização de conversas rápidas que abordam a percepção e perspectivas da família e do próprio paciente sobre seu cuidado e sua percepção sobre a assistência, pode trazer grandes benefícios não somente para a tomada de decisão, como também à reflexão e mudança comportamental do profissional médico. Rotinas dessa natureza podem abranger princípios como respeito e dignidade, pois, trazem informações sobre aspectos culturais, valores, crenças, encorajamento, colaboração e maior participação de familiares no cuidado ao paciente. A análise de resultados desta prática pode demonstrar como está a experiência do cliente em relação aos serviços oferecidos e medir resultados clínicos ${ }^{(19)}$.

A adaptação ao novo perfil do usuário de sistemas de saúde, que compreende o envelhecimento, exige a criação e oferta de serviços através de instituições de longa permanência, que podem garantir cuidados apropriados para pacientes em condições de doenças estáveis, porém, dependentes de auxílio e monitoramento ${ }^{(7)}$.

A oncologia integrativa procura engajar pacientes e familiares sobre a própria assistência e aplica cuidadosamente terapias complementares ao longo do tratamento convencional do câncer. Dessa forma, o paciente permanece no centro do cuidado e entende que não precisa optar entre duas linhas de tratamento, mas sim, pode recebê-los de forma integrada, melhorando sua qualidade de vida física, emocional e espiritual. A comunicação entre a equipe multiprofissional e o médico de referência à atenção primária é essencial para a efetividade desta abordagem ${ }^{(20)}$.

\section{CONCLUSÃO}

Um modelo assistencial adequado proporciona qualidade aos serviços e é fator determinante para a obtenção dos objetivos estratégicos e a proximidade com a carteira de beneficiários. É também fundamental para a perpetuação da relação da operadora com seus clientes, e favorece a aquisição das novas tecnologias de saúde, o adequado controle de sinistralidade, e por fim, a evolução e contribuição esperadas na assistência à saúde.

A proposta de modelo assistencial apresentada é indicada para inserir o paciente no centro do cuidado e buscar melhor experiência, valor e fidelidade do cliente, além de possibilitar o monitoramento do cuidado e a avaliação dos resultados operacionais. 


\section{REFERÊNCIAS}

1. Aidar MM, Burmester H. Planejamento Estratégico e Competitividade na Saúde (Série Gestão Estratégica de Saúde). São Paulo: Saraiva, 2015. 200p.

2. Organização Pan-Americana da Saúde. Brasil, Agência Nacional de Saúde Suplementar. Promoção da saúde e prevenção de riscos de doenças na saúde suplementar brasileira: Resultados do laboratório de inovação. Brasília, DF: OPAS, 2014.

3. Sikka R, Morath JM, Leape L. The Quadruple Aim: care, health, cost and meaning in work. BMJ Qual Saf 2015;0:1-3. https://doi.org/10.1136/bmjas$\underline{2015-004160 /}$

4. Agência Nacional de Saúde Suplementar (Brasil). Laboratórios de inovação sobre a experiência em atenção primária na saúde suplementar [recurso eletrônico] / Diretoria de Normas e Habilitação dos Produtos. Gerência Geral de Regulação Assistencial. Gerência de Monitoramento Assistencial, Organização Pan-americana da Saúde - Rio de Janeiro: ANS, 2018.

(NAVEGADORSUS; v.12) 2.3MB; ePUB.ISBN:1. Saúde Suplementar. 2 Atenção primária à saúde. I. Organização Pan-americana da Saúde.II.Título. Disponível em: http://www.ans.gov.br/biblioteca/index.html.

5. Malta DC, Felisbino-Mendes MS, Machado IE, Passos, VMA, Abreu DMX, Ishitani $\mathrm{LH}$, et al. Fatores de risco relacionados à carga global de doenças do Brasil e Unidades Federadas, 2015. Rev Bras de Epidemiologia, 20 Suppl 1:217-232, maio. 2017.

6. IPEA. Instituto de Pesquisa Econômica Aplicada. Desafios da Nação Volume 01. Brasília, 2018. Disponível em www.ipea.gov.br/portal/imagens/stories/PDFs/livros/livros/180327 desafios da nacao.pdf. Acesso em 25 dez 2019.

7. Mendes EV. O cuidado das condições crônicas na atenção primária à saúde: o imperativo da consolidação da estratégia da saúde da família. /Eugênio Vilaça Mendes. Brasília: Organização Pan - Americana da Saúde, 2012. 512p.iil. ISBN:978-85-7967-078-7.

8. Global Cancer Observatory (France). International Agency for Research on Cancer. World Health Organization. Disponível em: http://gco.iarc.fr. Acesso em: 3 Nov 2019.

9. Lopez G, MAO JJ, Cohen L. Integrative Oncology. Med Clin N Am. Volume 110, Issue 5 September 2017, pages 977-985. 
10. Minami B. Análise especial do mapa assistencial da saúde suplementar no Brasil entre 2013 e 2018. Disponível em:

http://www.iess.org.br/cms/rep/MApafinal.pdf. Acesso em: 8 dez.2019.

11. Strecker M. Planos de saúde enfrentam crises e procuram alternativas. Ago.2016. Disponível em:

https://www1.folha.uol.com.br/mercado/2016/08/1806351-planos-de-saudeenfrentam-crise-e-procuram-alternativas.shtml. Acesso em: 10 fev.2019.

12. Sá MR, Júnior JNM, Reinaldo LM. Processo de ruína finito: um estudo de caso na saúde suplementar no Brasil. Revista Evidenciação Contábil \& Finanças. João Pessoa, v.5, n2, p.88-103, mai./ago.2017. Disponível em: http://periódicos.ufpb.br/ojs2/index.php/recifn.

13. Colldén C, Gremyr I, Helström A, Sporraeus D.A value-based taxonomy of improvement approaches in healthcare. Journal of Health Organization and Management.V31, $n^{\circ} 4,2017$. pp. 445- 458.

14. Viana AL, Bousquat $A$, Melo GA, Filho AD, Medina MG. Regionalizacão e redes de Saúde. Ciência \& Saúde Coletiva. 2018; 23(6):1791-1798.

15. Oliveira MR, Veras RP, Cordeiro HA Pasinato MT. A mudança de modelo assistencial de cuidado ao idoso na Saúde Suplementar: identificação de seus pontos - chave e obstáculos para implementação. Physis vol.26. n .4. Rio de Janeiro. Oct/Dez .2016.

16. Neto GV, Malik, AM. Gestão em Saúde. Rio de Janeiro: Guanabara Koogan; 2011.

17. Lukosevicus AP, Guimarães JC. Uso do Método Estudo de Caso em Pesquisas de Gerenciamento de Projetos. Revista de Gestão e Projetos GeP. Vol. 9, N. 2. Maio / Agosto. 2018.

18. David G, Saynisch PA, McLallen, AS. The economics of patient - centered care. Journal of Health Economics. 59 (2018) 60 - 77. https://doi.org/10.1016/j.jhealeco.2018.02.012.

19. Fagan MB, Wong C, Carnie MB, Ashley SW, Somerville JG. Implementing Patient Family - Centered Care Grand Rounds Using Patient/Family Advisor Narratives. Journal of Patient Experience. Vol 2(2) 14-17. 2015.

20. Latte-Naor S, Mao JJ. Putting Integrative Oncology Into Practice: Concepts and Approaches. J Oncol Pract. 447-458. Out/Dez. 2009. n., v. 49. São Paulo. 
Recebido: 13 de maio de 2021. Aceito: 30 de junho de 2021

Correspondência: Alberto José Ogata. E-mail: alberto.ogata@fgv.br

Conflito de Interesses: o autor declarara não haver conflito de interesses

(C) This is an Open Access article distributed under the terms of the Creative Commons Attribution License, which permits unrestricted use, distribution, and reproduction in any medium, provided the original work is properly cited 